\title{
A phase I study on pharmacokinetics and pharmacodynamics of higenamine in healthy Chinese subjects
}

\author{
Sheng FENG ${ }^{1}$, Ji JIANG ${ }^{1}$, Pei HU ${ }^{1}$, Jian-yan ZHANG ${ }^{1}$, Tao LIU $^{1}$, Qian ZHAO ${ }^{1, *}$, Bi-lu LI \\ ${ }^{1}$ Clinical Pharmacology Research Center, Peking Union Medical College Hospital, Chinese Academy of Medical Science and Peking \\ Union Medical College, Beijing 100730, China; ${ }^{2}$ Zhuhai Rundu Pharmaceutical Co, Ltd, Guangzhou 510620, China
}

\begin{abstract}
Aim: To investigate the pharmacokinetics, pharmacodynamics, and safety of higenamine, an active ingredient of Aconite root, in healthy Chinese volunteers.

Methods: Ten subjects received continuous, intravenous infusion of higenamine at gradually escalating doses from 0.5 to 4.0 $\mu \mathrm{g} \cdot \mathrm{kg}^{-1} \cdot \mathrm{min}^{-1}$, each dose was given for $3 \mathrm{~min}$. Blood and urine samples were collected at designated time points to measure the concentrations of higenamine. Pharmacodynamics was assessed by measuring the subject's heart rate. A nonlinear mixed-effect modeling approach, using the software Phoenix NLME, was used to model the plasma concentration-time profiles and heart rate. Results: Peak concentrations $\left(C_{\max }\right)$ of higenamine ranged from 15.1 to $44.0 \mathrm{ng} / \mathrm{mL}$. The half-life of higenamine was $0.133 \mathrm{~h}$ (range, 0.107-0.166 h), while the area under concentration-time curve (AUC), extrapolated to infinity, was $5.39 \mathrm{ng} \cdot \mathrm{h} \cdot \mathrm{mL}^{-1}$ (range, $3.2-6.8$ $\mathrm{ng} \cdot \mathrm{h} \cdot \mathrm{mL}^{-1}$ ). The volume of distribution $(V)$ was $48 \mathrm{~L}$ (range, 30.8-80.6 L). The total clearance $(\mathrm{CL})$ was $249 \mathrm{~L} / \mathrm{h}(\mathrm{range}, 199-336$ $\mathrm{L} / \mathrm{h}$ ). Within $8 \mathrm{~h}, 9.3 \%$ (range, $4.6 \%-12.4 \%$ ) of higenamine was recovered in the urine. The pharmacokinetics of higenamine was successfully described using a two-compartment model with nonlinear clearance. In the pharmacodynamic model, heart rates were related to the plasma drug concentrations using a simple direct effect model with baseline. The $E_{0}, E_{\max }$, and $\mathrm{EC}_{50}$ were 68 bpm, 73 bpm and $8.1 \mu \mathrm{g} / \mathrm{L}$, respectively.

Conclusion: Higenamine has desirable pharmacokinetic and pharmacodynamic characteristics. The results provide important information for future clinical studies on higenamine.
\end{abstract}

Keywords: higenamine; Aconite root; coronary artery disease; stress myocardial perfusion imaging; pharmacologic stress test; pharmacokinetics; pharmacodynamics

Acta Pharmacologica Sinica (2012) 33: 1353-1358; doi: 10.1038/aps.2012.114; published online 22 Oct 2012

\section{Introduction}

Stress myocardial perfusion imaging is a widely used technique for the noninvasive evaluation of coronary artery disease (CAD), especially for patients who cannot exercise. Patients with coronary artery blockages may show minimal or no symptoms during rest. However, symptoms and signs of heart disease may be unmasked by exposing the heart to exercise- or drug-induced stress. Currently, the most popular drugs for pharmacologic stress tests are regadenoson, dipyridamole and dobutamine ${ }^{[1-3]}$, but these drugs commonly cause adverse effects and cannot be used in some patients ${ }^{[4-6]}$.

Higenamine is an active ingredient of Aconite root, which is used in Chinese herbal medicine (Figure 1). Higenamine has

\footnotetext{
* To whom correspondence should be addressed.

E-mail zhaoqian.pumch@gmail.com

Received 2012-05-09 Accepted 2012-07-13
}<smiles>Oc1ccc(CC2NCCc3cc(O)c(O)cc32)cc1</smiles>

Figure 1. Chemical structure of higenamine.

been shown to stimulate the cardiac $\beta$-adrenoceptor, vasodilation and anti-platelet aggregation through a-adrenoceptor interaction and could represent a new agent for pharmaceutical stress testing ${ }^{[7-11]}$. Moreover, higenamine was well tolerated among healthy Chinese subjects in a clinical trial, which reported that the highest safe dose was $24 \mu \mathrm{g} / \mathrm{kg}^{[12]}$.

To date, a pharmacokinetic and pharmacodynamic profile of 
higenamine has not been reported in humans. Here, we report the first clinical trial conducted to evaluate the pharmacokinetics and pharmacodynamics of higenamine in healthy Chinese subjects.

\section{Materials and methods Study design}

An open-label, non-controlled, single-dose, single-center study was conducted in healthy Chinese subjects. The study was approved by the Ethics Committee of Peking Union Medical College Hospital (PUMCH) and was accomplished in accordance with Good Clinical Practice guidelines. It conformed to the ethical principles of the Declaration of Helsinki. Written informed consent was obtained from each subject before the study.

\section{Subjects}

Subjects who were 19 to 45 years of age, had a body mass index (BMI) between 18 and $25 \mathrm{~kg} / \mathrm{m}^{2}$ and a body weight $>50$ $\mathrm{kg}$ (male) and $>45 \mathrm{~kg}$ (female) were eligible for inclusion in the study. Subjects with a recent history or the presence of any significant diseases, as judged by a medical interview and a physical examination, were not allowed to participate in the study. Additionally, subjects with any history of drug hypersensitivity, allergy or serious adverse drug reaction(s), including photosensitivity reaction, were excluded. For inclusion in the study, the results of clinical laboratory tests (including hematology, urinalysis and routine biochemistry) had to be within the normal range, and a normal baseline 12-lead electrocardiogram (ECG) was required for each subject. Subjects were excluded if they had participated in any other clinical trial or donated blood within $90 \mathrm{~d}$ prior to the beginning of this study. Subjects were also not allowed to participate in the study if their diastolic blood pressure was higher than $90 \mathrm{mmHg}$, their systolic blood pressure was higher than 140 $\mathrm{mmHg}$ or their heart rate was lower than 45 beats per minute (bpm) and/or higher than $90 \mathrm{bpm}$ while at rest.

\section{Methods and drug administration}

Subjects were admitted to the study center $24 \mathrm{~h}$ prior to administration of the drug on $\mathrm{d} 1$ and remained at the study center for $24 \mathrm{~h}$ after the treatment. During their stay at the study center, the subjects received continuous, intravenous infusion of higenamine at gradually escalating doses of $0.5 \mu \mathrm{g} \cdot \mathrm{kg}^{-1} \cdot \mathrm{min}^{-1}, 1.0 \mu \mathrm{g} \cdot \mathrm{kg}^{-1} \cdot \mathrm{min}^{-1}, 2.0 \mu \mathrm{g} \cdot \mathrm{kg}^{-1} \cdot \mathrm{min}^{-1}$ and 4.0 $\mu \mathrm{g} \cdot \mathrm{kg}^{-1} \cdot \mathrm{min}^{-1}$, each of which was given for $3 \mathrm{~min}$ (Figure 2). Standardized meals were provided at 3 and $9 \mathrm{~h}$ after treatment. Subjects had to abstain from vigorous physical activity, tobacco and alcohol consumption while they were at the study center.

\section{Sample collection and pharmacodynamic parameters}

On $\mathrm{d} 1$ of the study, blood samples were taken predose and at $3,6,9,12,13,15,17,19,21,24,27,32,42,72$, and $102 \mathrm{~min}$ postdose. These samples were used to determine the drug concentrations. Urine samples were collected over the follow-

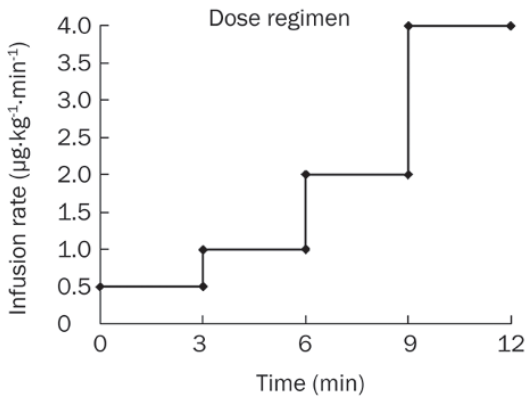

Figure 2. Dose regimen chart. Higenamine was intravenously infused at escalating doses, each of which was given for $3 \mathrm{~min}$.

ing time periods: predose, and $0-4$ and $4-8 \mathrm{~h}$ postdose. The urinary volumes were recorded, and $5 \mathrm{~mL}$ was used for the drug assays. All of the samples used for assays were stored at $-70^{\circ} \mathrm{C}$.

The heart rates were recorded predose and at 2, 5, 8, 11, 13, $15,17,27$, and $42 \mathrm{~min}$ postdose to assess the pharmacodynamics of higenamine.

\section{Safety analysis}

Safety measures included the collection of data on all adverse events, serious adverse events, clinical laboratory tests, vital signs and ECG parameters. Systolic and diastolic blood pressures were also recorded.

\section{Analytic assays}

The plasma and urine samples were analyzed by liquid chromatography-tandem mass spectrometry (LC-MS/MS) methods, which were fully validated according to the Standard Operation Procedure of PUMCH and Good Laboratory Practice guidelines. Higenamine and its internal standard, dobutamine, were extracted from a $0.20-\mathrm{mL}$ aliquot of plasma and urine by solid-phase extraction and injected into an API 4000 tandem MS (Applied Biosystems, CA, USA) and API 3000 tandem MS (Applied Biosystems, CA, USA), respectively. A $\mathrm{C}_{18}$ high-performance liquid chromatography column was used. The results were quantified by positive ionization mode with transition masses for higenamine and dobutamine of 272.1 to 107.1 and 302.2 to 137.1, respectively. The calibration curve ranged from $0.100-50.0 \mu \mathrm{g} / \mathrm{L}$ and $1.00-500 \mu \mathrm{g} / \mathrm{L}$ in plasma and urine, respectively. The precision of the assay was less than $15 \%$, as indicated by the coefficient of variation ( $\% \mathrm{CV})$, and the accuracy was within $\pm 15 \%$ of the actual concentration.

The heart rates were recorded using TM-2562P portable bedside monitors (A\&D, Tokyo, Japan).

\section{Pharmacokinetic and pharmacodynamic analysis}

First, the pharmacokinetic parameters were computed by noncompartmental analysis using Phoenix WinNonlin (version 6.1, Pharsight Corporation, CA, USA). The maximum observed plasma concentration $\left(C_{\max }\right)$, area under the concentration-time curve with extrapolation to infinity after a single dose $\left(\mathrm{AUC}_{0-\infty}\right)$, clearance $(\mathrm{CL})$, volume of distribution $(V)$, 
terminal half-life $\left(t_{1 / 2}\right)$, amount excreted in urine $\left(A_{\mathrm{e}}\right)$, fraction excreted in urine $(f e \%)$ and renal clearance $\left(\mathrm{CL}_{\mathrm{r}}\right)$ were calculated.

Second, a pooled dataset, including higenamine plasma concentration-time and heart rate-time data, were used for modeling. During the pharmacokinetic model-building procedure, different structural pharmacokinetic models were tested, including a one-compartment model, a two-compartment model and a three-compartment model with linear and nonlinear clearance. A two-compartment model with nonlinear clearance was chosen as the final model for the description of the plasma higenamine concentration-time course. In the pharmacodynamic model, individual heart rate values were directly related to higenamine plasma concentrations using a simple direct effect model with baseline: $E=E_{0}+E_{\max }{ }^{*} \mathrm{C} /$ $\left(\mathrm{EC}_{50}+\mathrm{C}\right)$, where $E, E_{0}, E_{\max }$ and $\mathrm{EC}_{50}$ are the heart rate, the baseline heart rate, the maximum increase in heart rate and plasma higenamine concentration causing a 50\% increase in the maximum heart rate, respectively.

Compartment analysis was performed by fitting a nonlinear mixed effects model using Phoenix NLME 1.0. The pharmacokinetic and pharmacodynamic parameters were estimated simultaneously using the first-order conditional estimationextended least squares (FOCE ELS) approach. The residual variability was investigated using an additive, multiplicative and mixed error model. A residual error model with a multiplicative component was applied in the final model.

Once the basic model had been selected, the effects of demographic factors on the model parameters were investigated graphically. The individual parameters obtained from the basic model by posterior conditional estimation technique were plotted separately against each covariate. The following covariates were examined: sex, height, weight, body mass index and age. A stepwise forward inclusion procedure $(P<0.05$; decrease in OFV $>3.84)$ was performed to build the full model, and a stepwise backward elimination procedure $(P<0.001$; increase in OFV $>10.83)$ was applied to determine the final model.

The minimum value of twice the negative log likelihood (-2LL) was used as a statistical method to choose suitable models during the model-building process.

\section{Results}

Subjects

Ten (4 male and 6 female) subjects were enrolled in this study. All subjects completed the trials as planned. The demographic details are presented in Table 1.

\section{Concentrations of higenamine in plasma and urine}

Following intravenous administration of $22.5 \mu \mathrm{g} / \mathrm{kg}$ of higenamine, the mean plasma concentration-time curve was calculated (Figure 3). The $A_{\mathrm{e}}$ and $f e \%$ were $120.6 \pm 34.5 \mu \mathrm{g}$ and $9.3 \% \pm 2.2 \%$, respectively.

\section{Noncompartmental pharmacokinetic analysis}

Pharmacokinetic parameters were calculated using the non-
Table 1. Subject demographics, mean \pm SD (range). $n=10$.

\begin{tabular}{ll}
\hline & Dose $22.5 \mu \mathrm{g} / \mathrm{kg}$ \\
\hline Age (year) & $30.2 \pm 6.8(22-41)$ \\
Sex & 4 male, 6 female \\
Height $(\mathrm{m})$ & $1.60 \pm 0.07(1.5-1.73)$ \\
Weight $(\mathrm{kg})$ & $60.4 \pm 4.2(52.5-66)$ \\
BMI* & $23.3 \pm 0.81(22.1-24.4)$ \\
\hline
\end{tabular}

BMI*: body mass index.

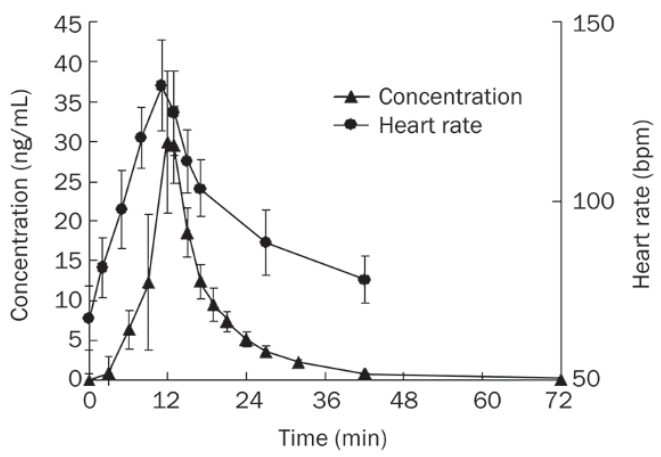

Figure 3. The average concentration-time curve of higenamine in the plasma and the heart rate-time curve from healthy Chinese subjects after intravenous administration of $22.5 \mu \mathrm{g} / \mathrm{kg}(n=10)$ higenamine hydrochloride (mean $\pm S D)$.

compartmental method, as follows. The $C_{\max }$ was obtained directly from the concentration-time data. The $C_{\max }$ for individuals ranged from 15.1 to $44.0 \mu \mathrm{g} / \mathrm{L}$. The $\mathrm{AUC}_{0-\infty}, V, \mathrm{CL}$, and $\mathrm{CL}_{\mathrm{r}}$ were $5.39 \mathrm{ng} \cdot \mathrm{h} \mathrm{mL}^{-1}$ (range, 3.2-6.8 $\mathrm{ng} \cdot \mathrm{h} \cdot \mathrm{mL}^{-1}$ ), $48 \mathrm{~L}$ (range, 30.8-80.6 L), $249 \mathrm{~L} / \mathrm{h}$ (range, 199-336 L/h) and 22.9 L/h (range, 12.9-29.4 L/h), respectively. The pharmacokinetic parameters are summarized in Table 2.

\section{Compartmental pharmacokinetic and pharmacodynamic analysis}

Individual plasma concentration-time profiles were accu-

Table 2. Noncompartmental pharmacokinetic parameters of higenamine after intravenous administration of $22.5 \mu \mathrm{g} / \mathrm{kg}(n=10)$ higenamine hydrochloride (mean $\pm S D$ ).

\begin{tabular}{lc}
\hline PK parameters & Dose $22.5 \mu \mathrm{g} / \mathrm{kg}$ \\
\hline$C_{\max }(\mu \mathrm{g} / \mathrm{L})$ & $31.3 \pm 9.24$ \\
$\mathrm{AUC} C_{\text {last }}\left(\mathrm{ng} \cdot \mathrm{h} \cdot \mathrm{mL}^{-1}\right)$ & $5.31 \pm 1.21$ \\
$\mathrm{AUC} \mathrm{C}_{0-\text { inf }}\left(\mathrm{ng} \cdot \mathrm{h} \cdot \mathrm{mL}^{-1}\right)$ & $5.39 \pm 1.23$ \\
$\mathrm{CL}(\mathrm{L} / \mathrm{h})$ & $249 \pm 42.78$ \\
$V(\mathrm{~L})$ & $48 \pm 13.83$ \\
$t_{1 / 2}(\mathrm{~h})$ & $0.133 \pm 0.02$ \\
$A_{\mathrm{e}}(\mu \mathrm{g})$ & $120.6 \pm 34.5$ \\
$\mathrm{CL}_{r}(\mathrm{~L} / \mathrm{h})$ & $22.9 \pm 4.41$ \\
$f e \%$ & $9.3 \pm 2.2$
\end{tabular}


rately described using the proposed two-compartment model with nonlinear clearance (Table 3 and Figure 4). The model parameters used are presented in Table 4. The proposed simple direct effect model with baseline was used to adequately describe the heart rate data, as evidenced by graphs of observed and predicted heart rates (Figure 5) and low CV values for the model parameters.

Table 3. Comparsion of different pharmacokinetic models.

\begin{tabular}{ll}
\hline \multicolumn{1}{c}{ PK models } & -2LL \\
\hline One-compartment model with linear clearance & 592 \\
One-compartment model with nonlinear clearance & 593 \\
Two-compartment model with linear clearance & 524 \\
Two-compartment model with nonlinear clearance & 510 \\
Three-compartment model with linear clearance & 524 \\
Three-compartment model with nonlinear clearance & 555 \\
\hline
\end{tabular}

$-2 L L=$ twice the negative log likelihood.
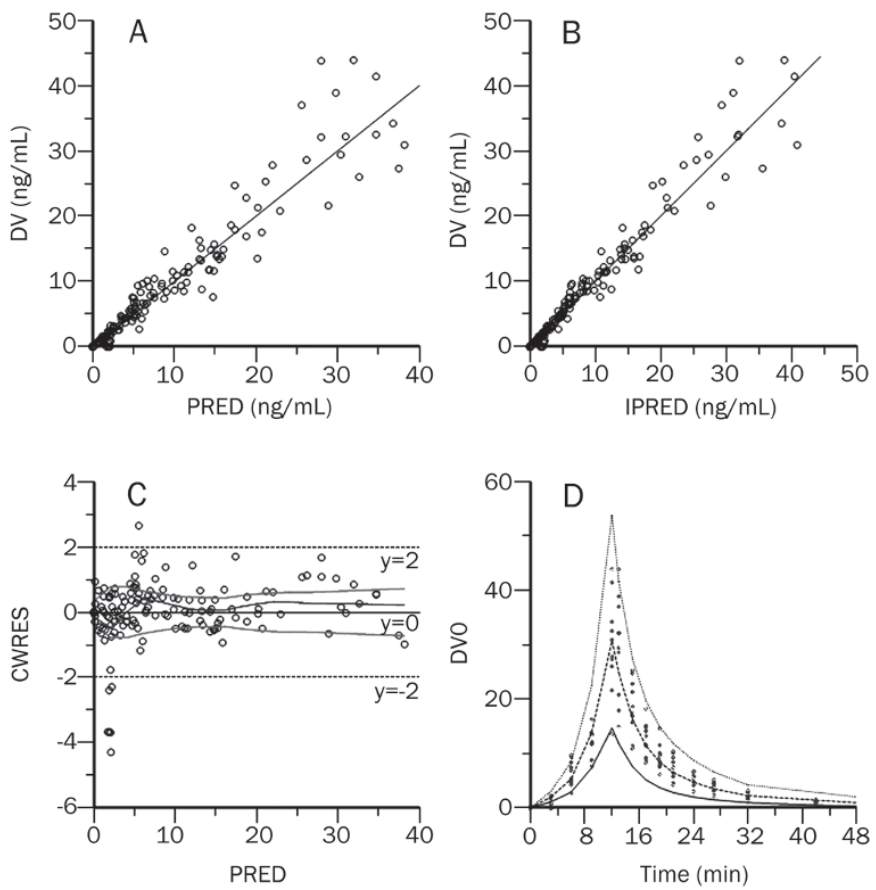

Figure 4. Observed data values (DV) vs population predicted data (PRED). (A) Individual predicted data (IPRED) and (B) Conditional Weighted Residual (CWRES) vs (C) population predicted data (PRED) and (D) Visual Predictive Check (VPC) profile of plasma concentration.

The effect of higenamine on the heart rate was rapid and evident. The subject heart rates increased in parallel with increasing doses of higenamine $\left(0.5\right.$ to $\left.4.0 \mu \mathrm{g} \cdot \mathrm{kg}^{-1} \cdot \mathrm{min}^{-1}\right)$. The mean heart rate-time curve is shown in Figure 3.
Table 4. Population pharmacokinetic and pharmacodynamic parameters of higenamine hydrochloride.

\begin{tabular}{|c|c|c|c|c|}
\hline \multirow[b]{2}{*}{ Parameter } & \multirow[b]{2}{*}{$\begin{array}{c}\text { Estimate } \\
(\% \mathrm{CV})\end{array}$} & \multirow{2}{*}{$\begin{array}{c}\text { Inter- } \\
\text { individual } \\
\text { variability } \\
(\% \mathrm{CV})\end{array}$} & \multicolumn{2}{|c|}{ Bootstrap } \\
\hline & & & $\begin{array}{l}\text { Median } \\
(\% \mathrm{CV})\end{array}$ & $\begin{array}{c}\text { Median } \\
\text { (2.5th, 97.5th } \\
\text { percentiles) }\end{array}$ \\
\hline$V_{c}(\mathrm{~L})$ & $18.7(6.6)$ & $8.7(4.1)$ & $19.1(17.5)$ & $14.0-27.0$ \\
\hline$V_{p}(\mathrm{~L})$ & $43.0(3.3)$ & & $45.9(25.5)$ & 25.1-71.4 \\
\hline$K_{m}(\mu g / L)$ & $3.1(3.7)$ & $4.1(0.3)$ & $3.6(89.1)$ & $0.635-12.3$ \\
\hline$V_{\max }(\mathrm{L} / \min )$ & $48.3(1.4)$ & $1.7(0.4)$ & 49.7 (37.5) & $28.0-100$. \\
\hline$C L_{d}(L / \min )$ & $3.8(5.2)$ & $1.0(0.05)$ & 3.7 (10.3) & $2.66-4.18$ \\
\hline \multicolumn{5}{|l|}{ Plasma } \\
\hline residual error & $0.260(6.1)$ & & $0.256(9.2)$ & $0.206-0.295$ \\
\hline$E_{0}(\mathrm{bpm})$ & $68(2.0)$ & $0.7(0.1)$ & $68(3.4)$ & 64-73 \\
\hline$E_{\max }(\mathrm{bpm})$ & $73(3.8)$ & $0.1(0.01)$ & $74(8.6)$ & $63-88$ \\
\hline $\mathrm{EC}_{50}(\mu \mathrm{g} / \mathrm{L})$ & $8.1(9.1)$ & & $8.5(31.0)$ & $4.4-14.6$ \\
\hline \multicolumn{5}{|l|}{ Heart rate } \\
\hline residual error & $0.089(8.1)$ & & $0.089(12.0)$ & $0.066-0.106$ \\
\hline
\end{tabular}

$V_{\mathrm{c}}=$ apparent volume of distribution of the central compartment; $V_{p}=$ apparent volume of distribution of the peripheral compartment; $K_{m}=$ Michaelis-Menten constant; $V_{\max }=$ maximal rate of metabolism; $C L_{d}=$ inter-compartmental clearance; $C V=$ coefficient of variance; $E_{0}=$ baseline heart rate; $\mathrm{EC}_{50}=$ concentration of drug producing $50 \%$ of maximum increase in heart rate; $E_{\max }=$ maximum increase in heart rate; bpm=beats per minute.
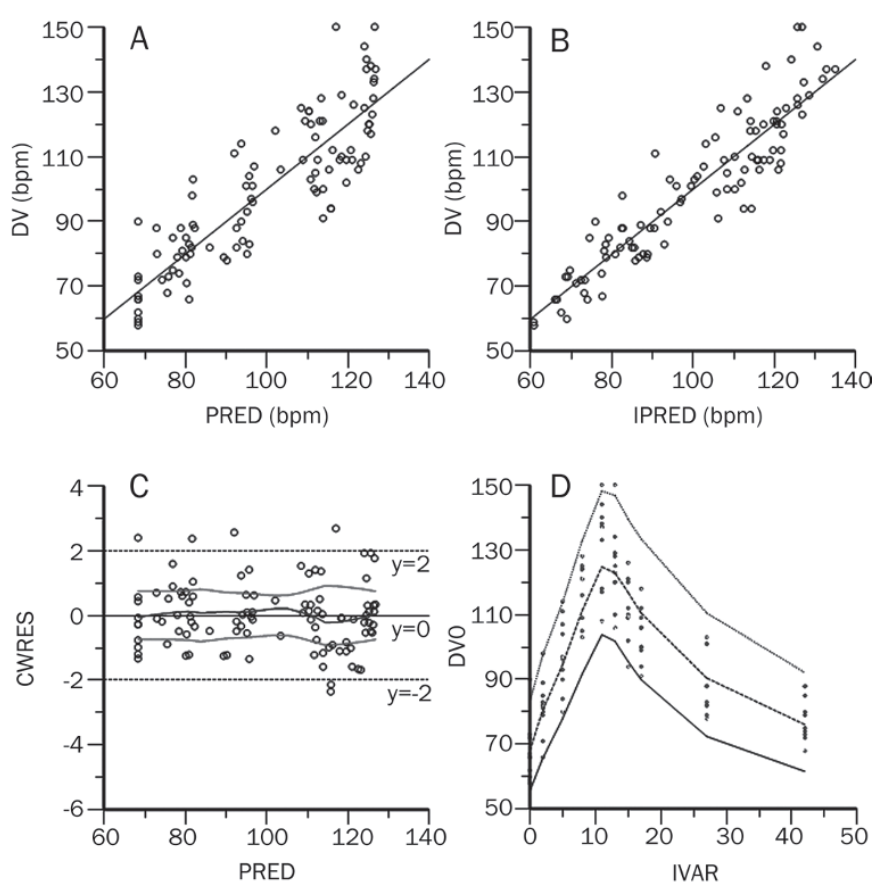

Figure 5. Observed data values (DV) vs population predicted data (PRED). (A) Individual predicted data (IPRED) and (B) Conditional Weighted Residual (CWRES) vs (C) population predicted data (PRED) and (D) Visual Predictive Check (VPC) profile of heart rate. 


\section{Model validation}

The accuracy and robustness of the final model were simultaneously evaluated using the resampling techniques of bootstrap and visual predictive check (VPC).

In this study, 1000 bootstrap samples were generated, and the population pharmacokinetic parameters were estimated for each of the 1000 samples using the final model. The mean and standard error of parameter estimates from the bootstrap analysis were then compared with the Phoenix NLME estimates from the final model (Table 4).

This final model adequately described the pharmacokinetic profile of higenamine, as shown by the VPC (Figure 4 and 5).

\section{Safety analysis}

Two of the 10 subjects reported 1 adverse event each. In one case, there was an increase of total bilirubin, which was mild and not considered related to the study drug. The other case subject reported dizziness and nausea, which was moderate and considered related to the study drug. Both of the adverse events were transient.

\section{Discussion}

Following intravenous administration of $22.5 \mu \mathrm{g} / \mathrm{kg}$ higenamine, the mean cumulative recovery of higenamine in the urine within $8 \mathrm{~h}$ was $9.3 \%$, which indicates that the kidney was not the major elimination route. The mean clearance and renal clearance were 249 and $22.9 \mathrm{~L} / \mathrm{h}$, respectively, which also suggest that the liver plays a more important role in the elimination of higenamine. While the highest hepatic blood flow was approximately $1.35 \mathrm{~L} / \mathrm{min}$ for a healthy subject during rest ${ }^{[13]}$, the non-renal clearance of higenamine was more than $220 \mathrm{~L} / \mathrm{h}$, indicating that the metabolism of higenamine needs to be further elucidated. The mean half-life of higenamine was $8.0 \mathrm{~min}$. Thirty minutes (approximately 4 half-lives) after administration, $94 \%$ of higenamine was eliminated from body. The heart rate of the subjects also returned to baseline $30 \mathrm{~min}$ after the infusion was completed. The fact that higenamine is quickly eliminated from the body is very helpful for the development of this agent for use in pharmaceutical stress testing.

An integrated pharmacokinetic and pharmacodynamic model of higenamine is necessary to address the relationship between exposure and response, and would provide potentially useful information for further clinical studies and higenamine pharmacotherapy. A simple direct effect with a baseline model, in which heart rate increased with increasing higenamine plasma concentrations, was used to accurately describe the pharmacokinetic and pharmacodynamic relationship. In response to higenamine, the heart rate increased rapidly, occurring within 2 min after drug administration. The $E_{\max }$ and $\mathrm{EC}_{50}$ were $73 \mathrm{bpm}$ and $8.1 \mu \mathrm{g} / \mathrm{L}$, respectively. For another stress test drug, regadenoson, which was approved by US Food and Drug Administration, a study was conducted on healthy male subjects in Scotland, and the $E_{\max }$ and $\mathrm{EC}_{50}$ were $76 \mathrm{bpm}$ and $12.3 \mu \mathrm{g} / \mathrm{L}$, respectively ${ }^{[14]}$. These results suggest that the pharmacologic stress effect of higenamine and regadenoson on the heart rate is similar.
At a dose of $22.5 \mu \mathrm{g} / \mathrm{kg}$, the average $C_{\max }$ of higenamine was $31.3 \mu \mathrm{g} / \mathrm{L}$. Based on the following equation, $E=E_{0}+E_{\max }{ }^{*} \mathrm{C} /$ $\left(\mathrm{EC}_{50}+\mathrm{C}\right)$, the average maximum heart rate was $126 \mathrm{bpm}$, which was less than $80 \%$ of the target heart rate $(220 \mathrm{bpm}-$ age). Thus, a higher dose of higenamine should be tested in future clinical studies. The analysis of pharmacokinetic data following administration of a higher dose of higenamine might also improve the estimation of saturable pharmacokinetic parameters.

In regard to the adverse effect related to the study drug, the $C_{\max }$ and $\mathrm{AUC}_{0-\infty}$ of the subject were $15.1 \mu \mathrm{g} / \mathrm{L}$ and 3.2 $\mathrm{ng} \cdot \mathrm{h} \cdot \mathrm{mL}^{-1}$, respectively, while the $C_{\max }$ and $\mathrm{AUC}_{0-\infty}$ from all subjects ranged from 15.1 to $44.0 \mu \mathrm{g} / \mathrm{L}$ and 3.2 to $6.8 \mathrm{ng} \cdot \mathrm{h} \cdot \mathrm{mL}^{-1}$, respectively. Therefore, the adverse effect was not related to exposure to the drug.

Higenamine did not cause a significant change in systolic blood pressure (the mean baseline was $109 \mathrm{mmHg}$, while the maximum was $130 \mathrm{mmHg}$ at $15 \mathrm{~min}$ postdose), but a slight decrease in diastolic blood pressure was observed (the mean baseline was $67 \mathrm{mmHg}$, while the minimum was $51 \mathrm{mmHg}$ at 11 min postdose).

Subject demographics were not found to influence the pharmacokinetic and pharmacodynamic profile of higenamine. However, it should be noted that only a few subjects with limited ranges of age, height and bodyweight values were included in this study; therefore, additional studies including older and heavier subjects should be considered.

In summary, intravenous administration of $22.5 \mu \mathrm{g} / \mathrm{kg}$ higenamine was well-tolerated in healthy Chinese volunteers. A two-compartment pharmacokinetic model was successfully fitted to the higenamine plasma concentration-time data, and a saturable pharmacodynamic model adequately described the increase in heart rate after drug administration.

\section{Acknowledgements}

This study was financially supported by the Institute of Materia Medica, the Cardiovascular Institute of the Chinese Academy of Medical Sciences \& Peking Union Medical College and by the Zhuhai Mingtong Medical Institute and Zhuhai Rundu Pharmaceutical Co, Ltd. This work was also supported by a grant from the National Program on Key Research Project of New Drug Innovation (№ 2008ZX09312-016).

\section{Author contribution}

Ji JIANG, Pei HU, Qian ZHAO, and Bi-lu LI designed this research; Jian-yan ZHANG and Sheng FENG performed this research; Sheng FENG and Tao LIU performed data analysis; Sheng FENG wrote the paper; and Ji JIANG, Pei HU, and Qian $\mathrm{ZHAO}$ critically revised the manuscript.

\section{References}

1 E Picano. Stress echocardiography from pathophysiological toy to diagnostic tool. Circulation 1992; 85: 1604-12.

2 Zoghbi GJ, Iskandrian AE. Selective adenosine agonists and myocardial perfusion imaging. J Nucl Cardiol 2012; 19: 126-41.

3 Elhendy A, Bax JJ, Poldermans D. Dobutamine stress myocardial 
perfusion imaging in coronary artery disease. J Nucl Med 2002; 43: 1634-46.

4 Barasch E, Wilansky S. Dobutamine stress echocardiography in clinical practice with a review of the recent literature. Tex Heart Inst J 1994; 21: 202-10.

5 Cortigiani L, Zanetti L, Bigi R, Desideri A, Fiorentini C, Nannini E. Safety and feasibility of dobutamine and dipyridamole stress echocardiography in hypertensive patients. J Hypertens 2002; 20: 1423-9.

6 Pingitore A, Picano E, Varga A, Gigli G, Cortigiani L, Previtali M, et al. Prognostic value of pharmacological stress echocardiography in patients with known or suspected coronary artery disease: a prospective, large-scale, multicenter, head-to-head comparison between dipyridamole and dobutamine test. Echo-Persantine International Cooperative (EPIC) and Echo-Dobutamine International Cooperative (EDIC) Study Groups. J Am Coll Cardiol 1999; 34: 1769-77.

7 Yun-Choi HS, Pyo MK, Park KM, Chang KC, Lee DH. Anti-thrombotic effects of higenamine. Planta Med 2001; 67: 619-22.

8 Chang KC, Chong WS, Lee IJ. Different pharmacological characteristics of structurally similar benzylisoquinoline analogs, papaverine, higenamine, and GS 389, on isolated rat aorta and heart. Can J
Physiol Pharmacol 1994; 72: 327-34.

9 Chong WS, Lee YS, Kang YJ, Lee DH. Comparison of inodilator effect of higenamine, YS49, YS51, tetrahydroisoquinoline analogs, and dobutamine in the rat. Korean J Physiol Pharmacol 1998; 2: 323-30.

10 Zhang Z, Liu X, Tao Z, Shi R, Zhang X, Yao Z, et al. Effects of higeramine on hemodynamics and its tolerability and safety, an experimental study. Zhonghua Yi Xue Za Zhi 2002; 82: 352-5.

11 Zheng YL, Shen R, Yang MF, Gu DL, Fang L, Zhang Z, et al. Experimental study of pharmaceutic stress myocardial perfusion imaging with higenamine. Zhonghua Xin Xue Guan Bing Za Zhi 2005; 33: 473-5.

12 Du YR, Li F, Xu RY, Zhang Y, Ouyang M, Jing HL. Tolerability of higenamine hydrochloride in healthy volunteers. Zhongguo Lin Chuang Yao Li Xue Za Zhi 2007; 23: 258-60.

13 Malcolm Rowland, Thomas N. Tozer. Clinical Pharmacokinetics and Pharmacodynamics: Concepts and Applications. 4th ed. Philadelphia: Lippincott Williams\&Wilkins; 2010.

14 Gordi T, Frohna P, Sun HL, Wolff A, Belardinelli L, Lieu H. A population pharmacokinetic/pharmacodynamic analysis of regadenoson, an adenosine $\mathrm{A}_{2 \mathrm{~A}}$-receptor agonist, in healthy male volunteers. Clin Pharmacokinet 2006; 45: 1201-12. 